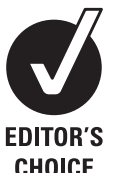

CHOICE

\title{
Six-month survival benefits associated with clinical guideline recommendations in acute coronary syndromes
}

\author{
D P Chew, ${ }^{1}$ F A Anderson, ${ }^{2}$ Á Avezum, ${ }^{3}$ K A Eagle, ${ }^{4}$ G FitzGerald, ${ }^{2} \mathrm{~J}$ M Gore, ${ }^{5}$ \\ $\mathrm{R}$ Dedrick, ${ }^{2} \mathrm{D}$ Brieger, ${ }^{6}$ for the GRACE Investigators
}

${ }^{1}$ Department of Cardiovascular Medicine, Flinders University, Adelaide, South Australia, Australia

${ }^{2}$ Center for Outcomes Research, University of Massachusetts Medical School, Boston, Massachusetts, USA

${ }^{3}$ Dante Pazzanese Institute of Cardiology, São Paulo, Brazil

${ }^{4}$ University of Michigan Health System, Ann Arbor, Michigan, USA

${ }^{5}$ Department of Cardiovascular Medicine, University of

Massachusetts Medical School, Boston, Massachusetts, USA ${ }^{6}$ Department of Cardiovascular Medicine, Concord Hospital, Sydney, New South Wales, Australia

\section{Correspondence to} Derek P Chew, Flinders Medical Centre, Flinders Drive, Bedford Park, SA 5042, Australia; derek.chew@flinders.edu.au

Accepted 18 February 2010 Published Online First 7 June 2010

\section{ABSTRACT}

Aims The authors sought to define which guidelineadvocated therapies are associated with the greatest benefit with respect to 6-month survival in patients hospitalised with an acute coronary syndrome (ACS).

Methods and results The authors conducted a nested case-control study of ACS patients within the Global Registry of Acute Coronary Events cohort between April 1999 and December 2007. The cases were ACS patients who survived to discharge but died within 6 months. The controls were patients who survived to 6 months, matched for ACS diagnosis, age and the Global Registry of Acute Coronary Events risk score. Rates of use of evidence-based medications and coronary interventions (angiography, percutaneous coronary intervention and coronary artery bypass graft surgery) were compared. Logistic regression including matched variables was used, and the attributable mortality from incomplete application of each therapy was calculated. A total of 1716 cases and 3432 controls were identified. Coronary artery bypass graft surgery and percutaneous coronary intervention were associated with the greatest 6-month survival benefit (OR for death $0.60(95 \% \mathrm{Cl} 0.39$ to 0.90) and 0.57 (0.48 to 0.72), respectively). Statins and clopidogrel provided the greatest independent pharmacologic benefit (ORs for death 0.85 (0.73 to 0.99) and 0.84 (0.72 to 0.99$))$ with lesser effects seen with other pharmacotherapies.

Conclusions A diminishing benefit associated with each additional ACS therapy is evident. These data may provide a rational basis for selecting between therapeutic options when compliance or cost is an issue.

Randomised clinical trials provide the evidence for modern clinical decision making. Consensus guidelines have become an important venue for translating trial data into routine care across a broad range of disease states, including acute coronary syndromes (ACSs). ${ }^{1-5}$ However, resulting from the manner by which this evidence is acquired, the relative clinical value of each new guideline-advocated therapy, added to and independent of other therapies, is uncertain. Consequently, with novel evidence, clinical guideline recommendations are often additive but do not inform us of therapies that may be omitted. When considering coronary revascularisation for instance, because access to this therapy is difficult in many parts of the world, establishing the relative advantages of invasive management in the context of more complete application of medical management may be useful for guiding resource allocation. The lack of data informing the choice between therapeutic strategies (eg, optimal revascularisation vs $\beta$-blockade) is a consequence of trial design, as randomised trials are directed at balancing clinical heterogeneity and are only able to optimally answer one question per randomisation. To date, several observational analyses have explored the cumulative value of guideline application, but not the specific contribution of each therapy. ${ }^{6} 7$ Furthermore, while clinical trials commonly advocate the application of all guidelinerecommended therapies, such application is rarely complete. This is particularly relevant when considering recommendations based on clinical trials conducted in an era predating modern cardiologic practice, such as the relative value of $\beta$-blockade in the post-revascularisation era. Hence, consideration of guidelines does not always inform us of the correct choice between therapeutic recommendations when choices have to be made. Nor does it provide data on the relative incremental benefit of one therapy over the application of all others. This is more of an issue when recommendations suggest the continuation of multiple therapies for an indefinite duration, in which case compliance becomes problematic.

We sought to explore the incremental gain associated with each of the current guideline-advocated therapies assuming that all others have already been optimally applied. To evaluate the impact of guideline-advocated therapies on 6-month survival in patients with ACS, we conducted a nested case-control study drawn from the Global Registry of Acute Coronary Events (GRACE) cohort to evaluate the impact of guideline-advocated therapies and treatment strategies on 6-month survival in patients with ACS.

\section{METHODS \\ Study population}

GRACE is designed to reflect an unselected population of patients with ACS, irrespective of geographic region. A total of 113 hospitals located in 14 countries in North and South America, Europe, Australia and New Zealand have contributed data to this study. Full details of the GRACE methods have been published elsewhere. ${ }^{8-10}$

Adult patients ( $>18$ years old) admitted with a presumptive diagnosis of ACS at participating hospitals were potentially eligible for this study. Eligibility criteria were a clinical history of ACS accompanied by at least one of the following: electrocardiographic changes consistent with ACS, serial increases in biochemical markers of cardiac necrosis (creatine kinase- $\mathrm{MB}$, creatine phosphokinase or troponin) and documented coronary artery disease. 
Patients with non-cardiovascular causes for the ACS clinical presentation, such as trauma or surgery, were excluded. The patients were followed-up at approximately 6 months by telephone, clinic visits or through calls to their primary care physician to ascertain the occurrence of several long-term outcomes. Where required, study investigators received approval from their local hospital ethics or institutional review board for the conduct of this study. Data were collected by trained study coordinators using standardised case report forms. Demographic characteristics, medical history, presenting symptoms, duration of prehospital delay, biochemical and electrocardiographic findings, treatment practises and a variety of hospital outcome data were collected. Standardised definitions of all patient-related variables, clinical diagnoses and hospital complications and outcomes were used. ${ }^{8}$ All the cases were assigned to one of the following categories: ST segment elevation myocardial infarction (STEMI), non-ST segment elevation myocardial infarction (NSTEMI) or unstable angina. Data from the patients enrolled between April 1999 and December 2007 were used in this analysis.

\section{Definition of cases and controls}

A nested matched case-control design was used because a large cohort of patients was available in the GRACE registry, enabling more explicit control of known powerful confounders, and such an analysis would be less impacted by issues of loss to follow-up. Cases were defined as patients presenting with ACS who survived to hospital discharge but died from any cause within 6 months of follow-up. All-cause mortality was used, as it is less subject to interpretation, whereas the duration of 6 months was used to enhance the likelihood that these events were cardiovascular in nature. Similarly, the patients presenting with an ACS who survived to 6 months were considered eligible controls. Standard definitions of ACS were used. The patients were with STEMI when they had new or presumed new ST segment elevation $\geq 1 \mathrm{~mm}$ seen in any location or new left bundle-branch block on the index or subsequent electrocardiogram with at least one positive cardiac biochemical marker of necrosis (including troponin measurements, whether qualitative or quantitative). In cases of NSTEMI, at least one positive cardiac biochemical marker of necrosis without new ST segment elevation seen on the index or subsequent electrocardiogram had to be present. Unstable angina was diagnosed when the serum biochemical markers indicative of myocardial necrosis in each hospital's laboratory were within the reference range.

\section{Matching}

Where possible, the cases and the controls were matched in a 1:2 ratio (cases to controls) based on the following factors: clinical diagnosis at discharge (STEMI, NSTEMI and unstable angina), age strata (grouped by 5 years) and GRACE risk score (grouped by five points). ${ }^{11}$ The patients with scores $<66$ (845 patients (2\%)) were excluded, as they were at very low risk, with only two deaths occurring in this group. Two controls were then found for each case.

\section{Therapies and treatment strategies}

We explored key guideline-recommended treatment strategies. ${ }^{3} 4$ Drug exposure was assessed by discharge prescriptions of aspirin, $\beta$-adrenoreceptor antagonists, angiotensin-converting enzyme (ACE) inhibitors or angiotensin receptor antagonists, clopidogrel and statins. Long-term compliance at 6 months after discharge was not assessed. Glycoprotein IIb/IIIa inhibitors were aggregated at the class level and identified if used at any time during hospitalisation. The following invasive procedures during index hospitalisation were recorded: coronary arteriography, percuta- neous coronary intervention (PCI) and/or coronary artery bypass grafting (CABG). PCI occurring as reperfusion for ST elevation (primary PCI) or as revascularisation were modelled as a single variable. Procedures after the initial hospitalisation or during subsequent hospitalisations were not included (ie, the patients were considered not to have received angiography or revascularisation) because these procedures occurred outside the exposure period of this study and may have been triggered by recurrent events. Referral to cardiac rehabilitation was identified by documentation of referral as recorded by the local site, with actual attendance and completion of the programme not confirmed.

\section{Statistical analysis}

All-cause mortality by 6 months was the dependent variable. Logistic regression including matched variables and all other potentially confounding variables $(p>0.2)$ was undertaken. Independent variables included the recommended therapies (aspirin, clopidogrel, glycoprotein IIb/IIIa inhibitors, angiotensinpathway blockers, $\beta$-adrenoreceptor antagonists, statins, arteriography, revascularisation and cardiac rehabilitation). These factors were considered independently and in conjunction with each other (revascularisation + statins + clopidogrel + rehabilitation + aspirin $+\beta$-adrenoreceptor antagonists + ACE inhibitor, etc). Other factors adjusted for in the model included the patient's country, diagnosis, cardiac arrest at presentation, Killip class and history of angina, myocardial infarction, heart failure, coronary artery disease, positive stress test, hypertension, dyslipidemia, LVEF, peripheral arterial disease, atrial fibrillation, transient ischaemic attack or stroke, diabetes, renal insufficiency, major surgery, venous thromboembolism, implantable defibrillators and major bleeding. A subanalysis confined to patients with STEMI was also conducted. In these models, the use of pharmacologic and catheter-based reperfusion was also assessed in conjunction with the other guideline recommendations.

Estimates from the logistic regression models and the rate of each therapeutic recommendation applied were used to estimate the adjusted attributable fraction (AF) of death preventable by the improved application of the clinical guidelines by calculating

$$
\mathrm{AF}=\frac{\text { Deaths }_{\mathrm{T}}-\text { Deaths }_{\mathrm{NG}}}{\text { Deaths }_{\mathrm{T}}}
$$

where Death $\mathrm{D}_{\mathrm{T}}$ is the total deaths predicted and Deaths $\mathrm{NG}_{\mathrm{N}}$ is the deaths occurring among people not receiving the guidelinerecommended therapy. ${ }^{12}$

Both the relative impact of each guideline (OR and 95\% confidence interval (CI)) and the adjusted absolute contribution (AF, percentage and 95\% CI) to 6-month survival were assessed for the entire population. The association between the number of guideline recommendations received (regardless of the specific guideline therapy given) and 6-month survival was assessed using logistic regression modelling and plotted in figure 1. In contrast, the predicted cumulative contribution of applying each of the guideline recommendations sequentially (greatest to least) was calculated by adding the $\beta$-coefficients for each of the recommendations in order and plotted in figure 2. For these estimates, PCI and CABG were combined into a single variable of revascularisation because these treatments are most often mutually exclusive. Continuous variables are expressed as a mean (SD) or median and interquatile range for variables with non-Gaussian distributions. All discrete variables are expressed as counts and percentages of the study population (n). The analyses were conducted using the SAS V.9 (SAS Institute) and the Stata 9.2 (Stata Corporation). A probability of $<0.05$ was considered statistically significant. 


\section{Number of therapies (vs. 0 or 1 therapy)}

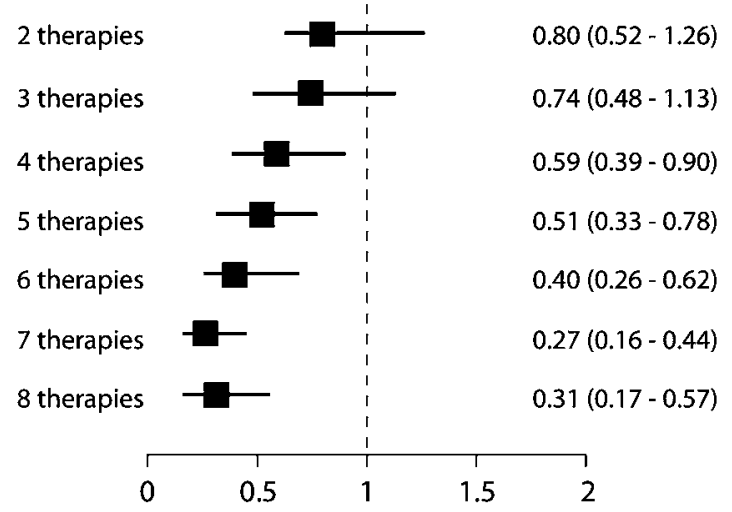

Figure 1 ORs for 6-month mortality associated with number of evidence-based guideline recommendations used.

\section{RESULTS}

Of the 41320 patients with GRACE risk scores who survived to hospital discharge, 1852 patients died by 6 months, and of these, 1716 cases (93\%) were identified as having appropriately matched controls $(n=3432)$. The characteristics of the study population are described in table 1 . The patients presenting with STEMI accounted for $37.2 \%$ of the overall population. Among several factors, patients dying within 6 months of hospital discharge were more likely to have a history of myocardial infarction and congestive heart failure and have hypertension, dyslipidemia and previous cerebrovascular disease.

\section{Use of guideline therapies}

The reported use of individual guideline recommendations among the cases and the controls is presented in table 2. Overall, patients surviving to 6 months were more likely to receive a greater number of guideline therapies. The adjusted ORs for mortality by 6 months with increasing guideline use are shown in figure 1.

\section{Guideline recommendations and 6-month survival}

The independent and relative relationship between the use of each guideline and 6-month survival is given in table 3. After adjustment for clinical and regional factors, undergoing PCI or CABG was associated with the most substantial relative survival advantage. Pharmacotherapies most associated with an improved survival at 6 months were clopidogrel and statins. The absolute contribution to total mortality (attributable risk, associated with incomplete implementation of clinical guidelines, or the amount of mortality that may be prevented with complete application of a given therapy) is presented in table 4. Up to $31.9 \%$ and $9.7 \%$ of deaths by 6 months may be prevented with more complete use of revascularisation and statin therapy, respectively.

\section{Combined effects of guidelines: STEMI patients}

In the smaller subset of patients with STEMI, the relationship between the guidelines was consistent with the analysis in the overall population. Apart from PCI, intravenous glycoprotein IIb/IIIa inhibition and statin therapy, many individual therapies did not reach statistical significance, contributed to by the smaller number of patients included. Fibrinolysis alone was not associated with reduced 6-month mortality after adjusting for other therapies (OR 0.96; 95\% CI 0.70 to $1.30 ; p=0.77$ ). When restricted to patients not receiving PCI (either as reperfusion or revascularisation), a significant benefit was observed (OR 0.74; 95\% CI 0.56 to $0.99 ; \mathrm{p}=0.04$ ).

\section{Combined effects of guidelines: all the patients}

When modelled collectively, application of all guideline recommendations in the overall population was associated with a lower 6-month mortality (OR 0.29 ; 95\% CI 0.19 to 0.44 ; $\mathrm{p}<0.001)$. In this analysis, little incremental gain in 6-month survival was observed with the application of more than six guideline recommendations, although there were wide confidence bounds around these estimates (figure 2).

\section{DISCUSSION}

With the expanding evidence base provided by clinical trials, the number of therapies and treatment strategies advocated by expert clinical guidelines can only increase. ${ }^{1-5}$ This increase places a burden on patients in terms of compliance and on health systems in terms of resource allocation. By weighing the relative and absolute value of each recommendation with respect to 6-month survival, in contrast with the other recommendations, this analysis may inform choice between therapies and strategies when such decisions need to be made. This analysis seeks to explore the relative mortality reduction associated with each guideline-recommended therapy after controlling for all others and describing the absolute proportion of lives that may be preserved with more complete application of each of the therapies. In this regard, coronary revascularisation appears to provide greater survival benefit than pharmacotherapy in relative terms. Among the pharmaceuticals, statin therapy and thienopyridines were associated with the greatest relative reductions in mortality. In absolute terms, more complete applications of revascularisation, statin therapy and clopidogrel have the greatest association with limiting the absolute numbers of lives lost by 6 months.

By the very nature of placebo-controlled clinical trial design, new evidence supporting novel treatment approaches provides evidence that is additive. Hence, these studies inform clinicians about the treatments that should be prescribed but rarely inform us of which therapies may be omitted. While the recent increase in non-inferiority studies provides some information regarding the choices between drugs, studies weighing the incremental

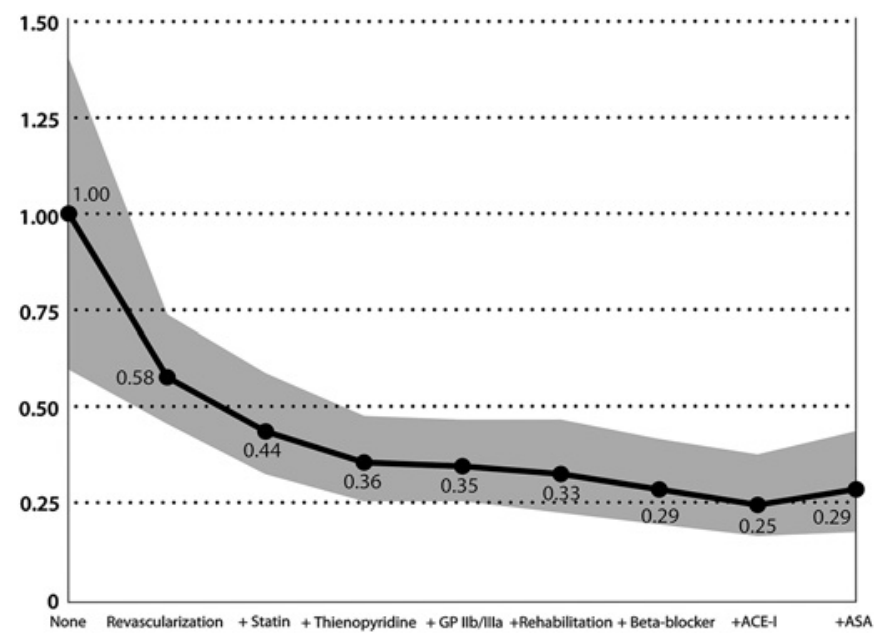

Figure 2 The mean effect of the sequential application of each guideline (greatest observed impact to least) and/or for 6-month mortality in patients with an ACS (PCI and CABG are considered revascularisation therapy). Cls are shown as the shaded area. 
Table 1 Baseline clinical and demographic characteristics

\begin{tabular}{|c|c|c|c|c|c|}
\hline & \multicolumn{2}{|c|}{$\begin{array}{l}\text { Cases } \\
(n=1716)\end{array}$} & \multicolumn{2}{|c|}{$\begin{array}{l}\text { Controls } \\
(n=3432)\end{array}$} & \multirow[b]{2}{*}{ p Value } \\
\hline & $\bar{n}$ & $\%$ & $\mathbf{n}$ & $\%$ & \\
\hline Female & 695 & 40.6 & 1401 & 41.0 & 0.83 \\
\hline Age, mean (SD) & 75.4 & (11.1) & 75.3 & (11.1) & 0.76 \\
\hline \multicolumn{6}{|l|}{ Diagnosis } \\
\hline $\begin{array}{l}\text { ST segment elevation myocardial } \\
\text { infarction }\end{array}$ & 639 & 37.2 & 1278 & 37.2 & 0.65 \\
\hline $\begin{array}{l}\text { Non-ST segment elevation } \\
\text { myocardial infarction }\end{array}$ & 744 & 43.4 & 1488 & 43.4 & \\
\hline Unstable angina & 333 & 19.4 & 666 & 19.4 & \\
\hline GRACE risk score, mean (SD) & 160.4 & (33.5) & 160.4 & $(33.6)$ & 1.00 \\
\hline \multicolumn{6}{|l|}{ Medical history } \\
\hline Angina & 961 & 56.2 & 1817 & 53.2 & 0.04 \\
\hline Myocardial infarction & 796 & 46.5 & 1145 & 33.5 & $<0.001$ \\
\hline Congestive heart failure & 518 & 30.4 & 585 & 17.1 & $<0.001$ \\
\hline $\begin{array}{l}\text { Angiogram diagnostic of coronary } \\
\text { artery disease }\end{array}$ & 629 & 37.6 & 962 & 28.5 & $<0.001$ \\
\hline $\mathrm{PCl}$ & 304 & 17.9 & 508 & 14.9 & 0.01 \\
\hline Coronary artery bypass graft & 304 & 17.8 & 442 & 12.9 & $<0.001$ \\
\hline Peripheral vascular revascularisation & 11 & 1.3 & 18 & 1.0 & 0.55 \\
\hline $\begin{array}{l}\text { Family history of coronary artery } \\
\text { disease }\end{array}$ & 176 & 21.2 & 405 & 22.8 & 0.36 \\
\hline Positive stress test & 184 & 10.9 & 314 & 9.2 & 0.06 \\
\hline Hypertension & 1231 & 72.1 & 2256 & 66.1 & $<0.001$ \\
\hline Dyslipidemia & 735 & 43.4 & 1434 & 42.2 & 0.42 \\
\hline Peripheral arterial disease & 305 & 17.9 & 419 & 12.3 & $<0.001$ \\
\hline Atrial fibrillation & 290 & 17.1 & 428 & 12.6 & $<0.001$ \\
\hline TIA/stroke & 289 & 17.0 & 393 & 11.5 & $<0.001$ \\
\hline Smoker & 923 & 54.0 & 1625 & 47.6 & $<0.001$ \\
\hline Former smoker & 604 & 66.4 & 1017 & 63.6 & \\
\hline Current smoker & 283 & 31.1 & 543 & 33.9 & 0.34 \\
\hline Status not recorded & 23 & 2.5 & 40 & 2.5 & \\
\hline Diabetes & 578 & 33.8 & 904 & 26.4 & $<0.001$ \\
\hline Diet controlled & 61 & 11.3 & 130 & 15.1 & \\
\hline Oral hypoglycemia & 244 & 45.0 & 443 & 51.3 & 0.004 \\
\hline Insulin dependent & 206 & 38.0 & 253 & 29.3 & \\
\hline No treatment used & 18 & 3.3 & 23 & 2.7 & \\
\hline Not recorded & 13 & 2.4 & 15 & 1.7 & \\
\hline Renal insufficiency & 327 & 19.2 & 379 & 11.1 & $<0.001$ \\
\hline No dialysis & 226 & 83.4 & 273 & 88.4 & 0.09 \\
\hline Dialysis & 45 & 16.6 & 36 & 11.7 & \\
\hline Major surgery/trauma & 159 & 9.3 & 203 & 5.9 & $<0.001$ \\
\hline Major bleeding & 47 & 2.8 & 79 & 2.3 & 0.34 \\
\hline Implantable cardiac defibrillator & 11 & 1.3 & 9 & 0.5 & 0.03 \\
\hline Venous thromboembolism & 33 & 3.9 & 43 & 2.4 & 0.03 \\
\hline \multicolumn{6}{|l|}{ Presentation/in-hospital } \\
\hline Cardiac arrest & 33 & 1.9 & 80 & 2.3 & 0.37 \\
\hline \multicolumn{6}{|l|}{ Killip class } \\
\hline I (no heart failure) & 1122 & 65.4 & 2320 & 67.6 & \\
\hline II (rales) & 428 & 24.9 & 752 & 21.9 & 0.09 \\
\hline III (pulmonary oedema) & 149 & 8.7 & 318 & 9.3 & \\
\hline IV (cardiogenic shock) & 17 & 1.0 & 42 & 1.2 & \\
\hline Left-ventricular ejection fraction & 1148 & 67.3 & 2399 & 70.5 & 0.02 \\
\hline Country & & & & & $<0.001$ \\
\hline Argentina & 99 & 5.8 & 263 & 7.7 & \\
\hline Australia/New Zealand & 172 & 10.0 & 354 & 10.3 & \\
\hline Austria & 15 & 0.9 & 43 & 1.3 & \\
\hline Belgium & 80 & 4.7 & 219 & 6.4 & \\
\hline Brazil & 132 & 7.7 & 214 & 6.2 & \\
\hline Canada & 92 & 5.4 & 238 & 6.9 & \\
\hline France & 100 & 5.8 & 279 & 8.1 & \\
\hline Germany & 58 & 3.4 & 119 & 3.5 & \\
\hline
\end{tabular}

Continued
Table 1 Continued

\begin{tabular}{|c|c|c|c|c|c|}
\hline & \multicolumn{2}{|c|}{$\begin{array}{l}\text { Cases } \\
(n=1716)\end{array}$} & \multicolumn{2}{|c|}{$\begin{array}{l}\text { Controls } \\
(\mathrm{n}=3432)\end{array}$} & \multirow[b]{2}{*}{ p Value } \\
\hline & $\bar{n}$ & $\%$ & $n$ & $\%$ & \\
\hline Italy & 22 & 1.3 & 100 & 2.9 & \\
\hline Poland & 77 & 4.5 & 213 & 6.2 & \\
\hline Spain & 62 & 3.6 & 206 & 6.0 & \\
\hline UK & 142 & 8.3 & 241 & 7.0 & \\
\hline USA & 665 & 38.8 & 943 & 27.5 & \\
\hline
\end{tabular}

value of well-established therapies in the modern era of ACS treatment are lacking. ${ }^{13}$ For example, clinical evidence supporting the long-term use of $\beta$-blockade among patients with ACS predates the current era where a relatively high rate of coronary angiography and revascularisation is practised. ${ }^{14}$ Hence, the incremental value of this guideline recommendation in the current context is unclear and is unlikely to be addressed in future randomised clinical trials. ${ }^{15}$

Consequently, within a nested case-control design, the impact of several therapeutic options can be weighted in the context of current clinical practise. With this analysis, estimates of the survival advantage with coronary revascularisation are greater than those observed in the clinical trials. ${ }^{16-19}$ This may be because patients enrolled in randomised trials may not be representative of individuals presenting in clinical practise. ${ }^{20}$ Our data are consistent with but extend beyond studies that support an early invasive approach to the management of ACS, although unmeasured biases cannot be excluded. These observations suggest that invasive management resulting in revascularisation does provide a reduction in mortality, reinforcing the clinical trial data that have relied upon composite ischaemic end points and that have at times been inconsistent. The benefits associated with pharmacotherapeutic drugs such as statins and clopidogrel are also consistent with key clinical trials. ${ }^{21-24}$ In contrast, the relationship with fibrinolysis is more modest than that observed in clinical trials and may be accounted for by PCI occurring both as reperfusion and revascularisation being included in the model. When the analysis was restricted to the patients not receiving any form of PCI, a benefit comparable with effects seen in clinical trials is evident. The modest benefits observed with the use of aspirin were less striking than expected and are likely explained by the high rate of use and, therefore, a low capacity to detect a difference associated with this therapy. Of course, all of these relative effects will be influenced by the adoption of new therapies into current practise.

At a clinical level, being able to independently value these therapies may provide the rationale for choosing between treatments when a choice must be made, either for reasons of cost or compliance. Among pharmacotherapies, statins and clopidogrel seem to impart the greatest additional benefit in this analysis. These drugs should, therefore, be the focus of efforts to improve compliance. Conversely, these data are reassuring when considering stopping $\beta$-blockade among patients who are poorly compliant or face significant adverse effects. Consequently, such an analysis may further the interpretation of performance measures in ACS care, which currently tend to weigh these measures either equally or cumulatively. ${ }^{25}$ However, such analyses cannot displace clinical judgement when considering any individual patient. Rather, they reflect potential gains at a population or average patient level.

At a policy level, these data help inform quality improvement priorities. Coronary revascularisation is associated with the greatest survival benefit, in part related to its incomplete application. Hence, limitations in the conduct of revascularisation 
Table 2 Rates of individual evidence-based recommendations among cases and controls

\begin{tabular}{|c|c|c|c|c|c|}
\hline & \multicolumn{2}{|c|}{$\begin{array}{l}\text { Cases } \\
(n=1716)\end{array}$} & \multicolumn{2}{|c|}{$\begin{array}{l}\text { Controls } \\
(\mathrm{n}=3432)\end{array}$} & \multirow[b]{2}{*}{ p Value } \\
\hline & $\mathbf{n}$ & $\%$ & $\mathbf{n}$ & $\%$ & \\
\hline Cardiac catheterisation & 692 & 40.9 & 1772 & 51.9 & $<0.001$ \\
\hline $\mathrm{PCl}$ & 329 & 19.3 & 1111 & 32.5 & $<0.001$ \\
\hline Coronary artery bypass graft surgery & 49 & 2.9 & 150 & 4.4 & 0.01 \\
\hline ACE inhibitor & 1148 & 67.4 & 2321 & 68.0 & 0.68 \\
\hline Aspirin & 1563 & 91.2 & 3194 & 93.1 & 0.02 \\
\hline$\beta$-Blocker & 1340 & 79.0 & 2775 & 81.3 & 0.05 \\
\hline Clopidogrel & 661 & 39.0 & 1705 & 50.0 & $<0.001$ \\
\hline Intravenous glycoprotein Ilb/llla inhibitor & 299 & 17.7 & 821 & 24.2 & $<0.001$ \\
\hline Statin & 951 & 55.7 & 2097 & 61.6 & $<0.001$ \\
\hline Referral to cardiac rehabilitation & 302 & 23.7 & 752 & 28.5 & 0.001 \\
\hline
\end{tabular}

represent the largest missed opportunity for preventing deaths by 6 months among patients with an ACS. ${ }^{26}$ However, these data are in contrast to evidence suggesting a lack of mortality benefit associated with access to hospital with onsite invasive services. ${ }^{27}$ This difference likely reflects the focus on provision of rather than the opportunity for revascularisation between these two studies. These observations lend themselves to the development of institutional performance indicators that reflect the rate of coronary angiography and revascularisation provision and inform the design of healthcare systems and referral patterns that support early access to invasive investigation and management.

\section{Limitations}

Several limitations should be considered. First, as with all observational studies, the possibility for unmeasured biases exists, thus leading to overestimation or underestimation of treatment effects. Specifically, there is the potential for selection of those

Table 3 Relationship between evidence-based recommendations and 6 -month survival in the overall population and among patients with STEMI

\begin{tabular}{|c|c|c|c|c|}
\hline & $\mathbf{O R}$ & \multicolumn{2}{|c|}{$95 \% \mathrm{Cl}$} & p Value \\
\hline \multicolumn{5}{|l|}{ Overall population } \\
\hline Coronary artery bypass graft surgery & 0.53 & 0.34 & 0.82 & 0.005 \\
\hline $\mathrm{PCl}$ & 0.63 & 0.48 & 0.81 & $<0.001$ \\
\hline Statin therapy & 0.74 & 0.63 & 0.88 & $<0.001$ \\
\hline Clopidogrel & 0.80 & 0.67 & 0.95 & 0.01 \\
\hline $\begin{array}{l}\text { Intravenous glycoprotein Ilb/Illa } \\
\text { inhibitor }\end{array}$ & 0.83 & 0.66 & 1.05 & 0.12 \\
\hline$\beta$-Blocker & 0.86 & 0.71 & 1.05 & 0.15 \\
\hline Referral to cardiac rehabilitation & 0.88 & 0.73 & 1.05 & 0.15 \\
\hline ACE inhibitor & 0.91 & 0.77 & 1.07 & 0.25 \\
\hline Aspirin & 0.97 & 0.73 & 1.29 & 0.83 \\
\hline Cardiac catheterisation & 1.14 & 0.92 & 1.40 & 0.23 \\
\hline \multicolumn{5}{|l|}{ Patients with STEMI } \\
\hline Coronary artery bypass graft & 0.53 & 0.25 & 1.10 & 0.09 \\
\hline $\mathrm{PCl}$ & 0.62 & 0.38 & 0.99 & 0.05 \\
\hline $\begin{array}{l}\text { Intravenous glycoprotein Ilb/llla } \\
\text { inhibitor }\end{array}$ & 0.63 & 0.43 & 0.92 & 0.02 \\
\hline Statin & 0.67 & 0.49 & 0.90 & 0.01 \\
\hline Clopidogrel & 0.78 & 0.57 & 1.08 & 0.14 \\
\hline$\beta$-Blocker & 0.83 & 0.58 & 1.19 & 0.32 \\
\hline Referral to cardiac rehabilitation & 0.84 & 0.62 & 1.14 & 0.26 \\
\hline ACE inhibitor & 0.95 & 0.70 & 1.29 & 0.76 \\
\hline Aspirin & 0.96 & 0.55 & 1.68 & 0.89 \\
\hline Fibrinolysis & 0.96 & 0.70 & 1.30 & 0.77 \\
\hline Cardiac catheterisation & 1.32 & 0.90 & 1.94 & 0.16 \\
\hline
\end{tabular}

Table 4 AF of 6-month mortality associated with incomplete application of evidence-based recommendations

\begin{tabular}{lcc}
\hline Guideline recommendation $^{*}$ & AF (\%) & $\mathbf{9 5 \%} \mathbf{C l}(\%)$ \\
\hline Revascularisation & 31.9 & 19.4 to 42.4 \\
Thienopyridine & 10.9 & 2.3 to 9.8 \\
Statin therapy & 9.7 & 4.1 to 15.0 \\
Rehabilitation referral & 10.6 & -2.4 to 21.5 \\
ACE inhibitor & 4.3 & -0.1 to 9.4 \\
Glycoprotein Ilb/lla inhibition & 1.9 & -16.8 to 17.3 \\
$\beta$-Blocker & 0.1 & -2.8 to 4.6 \\
\hline
\end{tabular}

*Effect of aspirin not estimatable.

controls with a greater likelihood for survival who are more likely to receive clinical guideline-recommended therapies and to be referred for cardiac rehabilitation. However, evidence suggests that biases towards lack of invasive management are coupled with underuse of guideline medications and interpreting the relative impact among therapies is less subject to this bias. Similarly, the case definition using all-cause mortality as opposed to cardiovascular mortality may bias towards a greater benefit seen with revascularisation but attenuate the observed impact of pharmacotherapies. Since angiography alone was not associated with any survival advantage, such biases would appear to be minimal, at least with respect to delivering invasive management, and it is the decision to undertake invasive management that is most likely coassociated with perceived survival.

Second, given that this analysis measures exposure to a guideline recommendation at a single time point (hospital discharge), bias towards revascularisation may exist. As ongoing adherence with pharmacotherapies is not measured, nonadherence may attenuate the benefit observed with drug therapies. Nevertheless, this is a persistent clinical problem compromising the effectiveness of all long-term therapeutic strategies. Adjustment for or exclusion of patients based on non-adherence would provide a false impression of true clinical effectiveness of these drugs. Third, the focus on patients that have survived to discharge may also provide a bias in favour of revascularisation because early mortality associated with revascularisation will not be included. However, inclusion of inhospital deaths would confound the analysis because it is also unlikely that the pharmacotherapies (apart from fibrinolysis) are likely to impact these early events. Last, the ability to confidently estimate the benefits of therapies that are close to completely applied (or very rarely used) such as aspirin and $\beta$-blockade is limited in this approach because of a lack of power, resulting from either relatively small numbers of people not receiving these therapies.

\section{Conclusions}

Within the modern management of patients presenting with ACS, a broad array of therapies are available and are recommended in clinical guidelines. Among these options, coronary revascularisation seems to provide the most robust survival advantage, highlighting the importance of improved systems of care enabling greater access to invasive management in many parts of the world. Among pharmacotherapies, statins and clopidogrel are associated with the greatest benefit. These data may provide a rational basis for selecting between therapeutic options when compliance or cost is an issue.

Acknowledgements The authors thank the physicians and nurses participating in the GRACE Study. Sophie Rushton-Smith, PhD, provided editorial support and was funded by sanofi-aventis. The GRACE Study is supported by an unrestricted 
educational grant from sanofi-aventis, Bridgewater, Massachusetts, USA, to the Center for Outcomes Research, University of Massachusetts Medical School. Sanofi-aventis had no involvement in the collection, analysis and interpretation of data, in the writing of the report and in the decision to submit the paper for publication.

Competing interests DPC receives funding from sanofi-aventis Australia and MSD Australia. KAE receives funding from Biosite, Bristol Myers Squibb, Cardiac Sciences, Blue Cross Blue Shield of Michigan, Hewlett Foundation, Mardigian Fund, Pfizer, sanofi-aventis, Varbedian fund, National Heart, Lung and Blood NIH. FAA, ÁA, JMG, $\mathrm{DB}$ and $\mathrm{RD}$ receive funding from sanofi-aventis.

Ethics approval The GRACE Study was conducted with the approval of each of the participating centres around the world.

Contributors Study concept and design: Chew

Acquisition of data: Chew, Avezum, Eagle, Gore and Brieger

Drafting of the manuscript: Chew and Brieger

Critical revision of the manuscript for important intellectual content: Chew, Anderson, Avezum, Eagle, Gore, FitzGerald and Brieger

Statistical analysis: FitzGerald and Dedrick

Administrative, technical or material support: Anderson.

Provenance and peer review Not commissioned; externally peer reviewed.

\section{REFERENCES}

1. Van de Werf F, Ardissino D, Betriu A, et al. Management of acute myocardial infarction in patients presenting with ST-segment elevation. the task force on the management of acute myocardial infarction of the European society of cardiology. Eur Heart J 2003;24:28-66.

2. Bertrand ME, Simoons ML, Fox KAA, et al. Management of acute coronary syndromes in patients presenting without persistent ST-segment elevation. Eur Heart J 2002;23:1809-40.

3. Kushner FG, Hand M, Smith SC Jr, et al. 2009 Focused updates: ACC/AHA guidelines for the management of patients with ST-elevation myocardial infarction (updating the 2004 guideline and 2007 focused update) and ACC/AHA/SCAI guidelines on percutaneous coronary intervention (updating the 2005 guideline and 2007 focused update): a report of the American College of Cardiology Foundation/ American Heart Association Task Force on Practice Guidelines. Circulation 2009; 120:2271-306

4. Anderson JL, Adams CD, Antman EM, et al. ACC/AHA 2007 guidelines for the management of patients with unstable angina/non ST-elevation myocardial infarction: a report of the American College of Cardiology/American heart association task force on practice guidelines (writing committee to revise the 2002 guidelines for the management of patients with unstable angina/non-ST-elevation myocardia infarction): developed in collaboration with the American College of Emergency Physicians, the Society for Cardiovascular Angiography and Interventions, and the Society of Thoracic Surgeons: endorsed by the American Association of Cardiovascular and Pulmonary Rehabilitation and the Society for Academic Emergency Medicine. Circulation 2007:116:e148-304.

5. Aroney CN, Aylward P, Kelly AM, et al. National Heart Foundation of Australia Cardiac Society of Australia and New Zealand Guidelines for the management of acute coronary syndromes 2006. Med J Aust 2006;184:S1-30.

6. Alexander KP, Roe MT, Chen AY, et al. Evolution in cardiovascular care for elderly patients with non-ST-segment elevation acute coronary syndromes: results from the CRUSADE National quality improvement initiative. J Am Coll Cardiol 2005:46:1479-87.

7. Mukherjee D, Fang J, Chetcuti S, et al. Impact of combination evidence-based medical therapy on mortality in patients with acute coronary syndromes. Circulation 2004;109:745-9.
8. Anon. Rationale and design of the GRACE (Global Registry of Acute Coronary Events) Project: a multinational registry of patients hospitalized with acute coronary syndromes. Am Heart J 2001:141:190-9.

9. Eagle KA, Goodman SG, Avezum A, et al. Practice variation and missed opportunities for reperfusion in ST-segment-elevation myocardial infarction: findings from the Global Registry of Acute Coronary Events (GRACE). Lancet 2002;359:373-7.

10. Steg PG, Goldberg RJ, Gore JM, et al. Baseline characteristics, management practices, and in-hospital outcomes of patients hospitalized with acute coronary syndromes in the Global Registry of Acute Coronary Events (GRACE). Am J Cardiol 2002:90:358-63

11. Granger CB, Goldberg RJ, Dabbous 0, et al. Predictors of hospital mortality in the global registry of acute coronary events. Arch Intern Med 2003;163:2345-53.

12. Benichou J. Methods of adjustment for estimating the attributable risk in case-control studies: a review. Stat Med 1991:10:1753-73.

13. Stone GW, McLaurin BT, Cox DA, et al. Bivalirudin for patients with acute coronary syndromes. N Engl J Med 2006;355:2203-16.

14. Anon. The beta-blocker heart attack trial. beta-Blocker Heart Attack Study Group. JAMA 1981;246:2073-4.

15. Chen ZM, Pan HC, Chen YP, et al. Early intravenous then oral metoprolol in 45,852 patients with acute myocardial infarction: randomised placebo-controlled trial. Lancet 2005;366:1622-32

16. Bavry AA, Kumbhani DJ, Rassi AN, et al. Benefit of early invasive therapy in acute coronary syndromes: a meta-analysis of contemporary randomized clinical trials. J Am Coll Cardiol 2006;48:1319-25.

17. Cannon CP, Weintraub WS, Demopoulos LA, et al. Comparison of early invasive and conservative strategies in patients with unstable coronary syndromes treated with the glycoprotein Ilb/lla inhibitor tirofiban. N Engl J Med 2001;344:1879-87.

18. de Winter RJ, Windhausen $\mathrm{F}$, Cornel $\mathbf{J H}$, et al. Early invasive versus selectively invasive management for acute coronary syndromes. $N$ Engl J Med 2005;353:1095-104.

19. Fox KA, Poole-Wilson PA, Henderson RA, et al. Interventional versus conservative treatment for patients with unstable angina or non-ST-elevation myocardia infarction: the British Heart Foundation RITA 3 randomised trial. Randomized Intervention Trial of unstable angina. Lancet 2002;360:743-51.

20. Steg PG, Lopez-Sendon J, Lopez de Sa E, et al. External validity of clinical trials in acute myocardial infarction. Arch Intern Med 2007;167:68-73.

21. Yusuf S, Zhao F, Mehta SR, et al. Effects of clopidogrel in addition to aspirin in patients with acute coronary syndromes without ST-segment elevation. N Engl J Med 2001;345:494-502.

22. Sabatine MS, Cannon CP, Gibson CM, et al. Effect of clopidogrel pretreatment before percutaneous coronary intervention in patients with ST-elevation myocardial infarction treated with fibrinolytics: the PCI-CLARITY study. JAMA 2005;294:1224-32.

23. Cannon CP, Steinberg BA, Murphy SA, et al. Meta-analysis of cardiovascular outcomes trials comparing intensive versus moderate statin therapy. J Am Coll Cardiol 2006:48:438-45.

24. Hulten E, Jackson JL, Douglas K, et al. The effect of early, intensive statin therapy on acute coronary syndrome: a meta-analysis of randomized controlled trials. Arch Intern Med 2006:166:1814-21.

25. Peterson ED, Roe MT, Lytle BL. The association between care and outcomes in patients with acute coronary syndrome: national results from CRUSADE (abstract) J Am Coll Cardiol 2004;43:406A.

26. Bhatt DL, Roe MT, Peterson ED, et al. Utilization of early invasive management strategies for high-risk patients with non-ST-segment elevation acute coronary syndromes: results from the CRUSADE Quality Improvement Initiative. JAMA 2004;292:2096-104.

27. Van de Werf F, Gore JM, Avezum A, et al. Access to catheterisation facilities in patients admitted with acute coronary syndrome: multinational registry study. $B M$ 2005;330:441. 